\title{
Wedelolactone Targets EZH2-mediated Histone H3K27 Methylation in Mantle Cell Lymphoma
}

\author{
NADEZHDA ROMANCHIKOVA and PETERIS TRAPENCIERIS
}

Latvian Institute of Organic Synthesis, Riga, Latvia

\begin{abstract}
Background/Aim: Enhancer of zeste homolog 2 (EZH2), the catalytic subunit of polycomb repressive complex 2 (PRC2), possesses histone N-methyltransferase (HMT) activity and plays an essential role in cancer initiation and development. The aim of the present study was to investigate the potential of Wedelolactone (WL) to inhibit the methylation activity of EZH2. Materials and Methods: The mantle cell lymphoma (MCL) cell line, Mino, was treated with WL, while untreated cells were used as control. HMT activity and EZH2 amount were measured in nuclear extracts from WL-treated and control Mino cells. Results: WL was found to target EZH2-mediated histone H3K27 methylation. Along with the inhibition of H3K27 methylation in vitro $(I C 50=0.3 \mu M)$, WL suppressed HMT activity in Mino cells with an IC50 value of $3.2 \mu \mathrm{M}$. We detected a reduced amount of EZH2 in Mino cells treated with WL, compared to untreated control cells. Conclusion: This is the first study to show that WL induces inhibition of $H 3 K 27$ methylation via EZH2 modulation and decreases cell proliferation in $M C L$, in vitro. WL is proposed as a promising agent and a novel epigenetic approach in $M C L$ investigation and treatment.
\end{abstract}

Recent investigations have revealed the involvement of multifunctional protein complexes in the regulation of cell malignancies. In the last few years much attention was paid to association of polycomb repressive complex 2 (PRC2) with cancer progress (1-3). EZH2, the enzymatic unit of the PRC2 complex, catalyzes the transfer of 1-3 methyl groups from S-adenozyl-L-methionine to the lysine residues of histone H3 (4). The tri-methylated lysine 27 of histone H3 (H3K27) has been shown as a stable facultative heterochromatin mark which

Correspondence to: Nadezhda Romanchikova, Latvian Institute of Organic Synthesis, Aizkraukles Str. 21, LV-1006, Riga, Latvia. Tel: +371 66155119, Fax: +371 66155119, e-mail: nadezhda romanchikova@gmail.com

Key Words: EZH2, H3K27 methylation, mantle cell lymphoma, wedelolactone, PRC2. promotes the recruitment of polycomb group proteins for promoter-targeted transcriptional gene silencing (5). Increased histone H3K27 methylation has been found to be involved in some pathological processes, especially cancer progress.

It has been shown that EZH2 is frequently overexpressed in many cancer types including lymphomas, breast, prostate, lung, brain, and liver cancer, and moreover, it is critical for cancer cell proliferation (6-10). Few reports have proposed mechanisms by which the PRC2 complex controlled methylation level. For example, it was shown that embryonic ectoderm development (EED) component of PRC2 is required for the histone $\mathrm{H} 3$ lysine methylation (11-13). Several natural compounds, such as curcumin, gambogic acid, triptolide, wedelolactone, and ursolic acid, have been shown to serve as EZH2 modulators (14), but their mechanism of action is poorly studied. Chen and co-authors demonstrated that wedelolactone (WL) binds with a high affinity to the EED and blocks the EZH2-EED interaction in vitro, inducing the degradation of PRC2 core components (15). WL has been used as an inhibitor of IkB kinase, which is critical for the activation of NF-kB survival pathway (16), and was shown to inhibit proliferation of prostate cancer cells in vitro and in vivo (17). In addition, WL has been found to reduce the production of interleukin- 6 in pancreatic cancer cells, which is responsible for their survival and proliferation (18). Moreover, WL induced apoptosis and prevented osteolytic bone metastasis in patients with bone metastasis of breast cancer origin $(19,20)$.

Mantle cell lymphoma (MCL) is a well-defined and aggressive type of B cell non-Hodgkin's lymphoma. Although drugs such as ibrutinib have shown promising therapeutic effect in clinical trials, MCL patients often acquired drug resistance (21). Therefore, new therapeutic approaches for MCL treatment are required. In this study we focused on the effects of WL on histone methylation mediated by the catalytic subunit EZH2 of PRC2 complex, in MCL cells.

\section{Materials and Methods}

Cell lines and cell culture. The MCL (non-Hodgkin's B-cell lymphoma) cell line, Mino, was obtained from American Type Cell 
Culture Collection (ATCC, CRL-3000; Manassas, VA, USA). Mino cells were maintained in RPMI 1640 medium supplemented with $2 \mathrm{mM}$ L-glutamine and 15\% fetal bovine serum (all from Sigma-Aldrich, St. Louis, $\mathrm{MO}, \mathrm{USA}$ ), at $37^{\circ} \mathrm{C}$ in a $5 \% \mathrm{CO}_{2}$ humidified atmosphere.

Cell treatment with WL. WL was purchased from TargetMol Corp. (Boston, MA, USA) and was dissolved in dimethyl sulfoxide as 100 $\mathrm{mM}$ stock solution. Mino cells were treated with the appropriate concentration of WL for indicated time periods, while untreated cells were used as control.

WST-8 cell proliferation assay. To evaluate WL cytotoxicity, WST8 assays (Cayman Chemicals, Ann Arbor, MI, USA) were performed according to manufacturer's protocol, as it was already described (22). All data are given as the mean of three independent experiments \pm standard deviation $(\mathrm{SD})$.

Nuclear proteins extraction. The kit from Abcam (Cambridge, UK) was used for extracting nuclear proteins from Mino cells, according to manufacturer's protocol. Mino cells $\left(5 \times 10^{6} / \mathrm{ml}\right)$ were treated with/without the appropriate concentration of WL, for indicated time periods. The concentration of nuclear proteins was measured at optical density $595 \mathrm{~nm}\left(\mathrm{OD}_{595}\right)$ using Bradford reagent (Abcam, Cambridge, UK), according manufacturer's protocol and was calculated using bovine serum albumin standard curve. All data are given as the mean of three independent experiments $\pm \mathrm{SD}$.

Histone H3K27 methyltransferase activity assay. The effect of WL on HMT activity was investigated by two ways. First, HMT activity was measured in NEs from untreated Mino cells, after direct exposure to WL for $1 \mathrm{~h}$ at concentrations of $0.1 \mu \mathrm{M}, 1.0 \mu \mathrm{M}$ and $10.0 \mu \mathrm{M}$. Second, HMT activity was measured in NEs from cells treated for $24 \mathrm{~h}$ with WL at concentrations of $0.1 \mu \mathrm{M}, 1.0 \mu \mathrm{M}, 3.0 \mu \mathrm{M}$ and $7.5 \mu \mathrm{M}$. In both experiments, $\mathrm{NE}$ from untreated cells, not further exposed to WL was used as control (C). The Histone H3 (K27) Methyltransferase Activity Quantification Assay kit (Abcam, Cambridge, UK) was used according to the manufacturer's instructions. Briefly, in a 96-well plate coated with histone $\mathrm{H} 3$ substrate, nuclear extracts (NEs) were added in each well. H3K27 histone methyltransferase (HMT) activity was detected with an anti-trimethyl-H3K27 specific antibody followed by a HRPconjugated secondary antibody. Color development was measured at $\mathrm{OD}_{450}$ and the HMT activity was quantified in $W L$-treated/untreated cells using the formula: $O D / h / m g=\left(\right.$ treated/untreated $N E O D_{450}$ blank $\left.O D_{450}\right) * 1000 / N E(\mu g) \times$ time $(h)$. HMT activity in untreated cells (C) was considered as $100 \%$ activity. All data are given as the mean of four independent experiments $\pm \mathrm{SD}$.

H3K27 methylation activity of EZH2 in the PRC2 complex, in vitro. Experiments were performed using the EZH2 Chemiluminescent Assay Kit (BPS Bioscience, San Diego, CA, USA) according to manufacturer's protocol, which measures the methylation activity of the PRC2 complex (EZH2/EED/SUZ12/RbAp48/AEBP) or reduced (EZH2/EED/SUZ12) PRC2 complex. The 96-well plate was precoated with $\mathrm{H} 3 \mathrm{~K} 27$ substrate, S-adenosyl- methionine was incubated with EZH2 $(10 \mathrm{ng} / \mu \mathrm{l})$ with WL or without WL in control. Primary antibody against methylated H3K27 and HRP-labeled secondary antibody followed by addition substrate to produce chemiluminescence were used. Luminescence in untreated wells was taken as $100 \%$ activity. All data are given as the mean of four independent experiments \pm SD.
Table I. Histone methyltransferase (HMT) activity specific for H3K27 methylation and cell proliferation, were measured in Mino cells treated with Wedelolactone for $24 \mathrm{~h}$ compared to untreated cells in the control (C). The data are presented as mean $\pm S D$.

\begin{tabular}{lccc}
\hline $\begin{array}{l}\text { Wedelolactone } \\
(\mu \mathrm{M})\end{array}$ & \multicolumn{2}{c}{ HMT activity } & $\begin{array}{c}\text { Alive cells } \\
\%\end{array}$ \\
\cline { 2 - 3 } & $\mathrm{OD} / \mathrm{h} / \mathrm{mg}$ & $\%$ & \\
\hline & & & \\
$\mathrm{C}$ & $71.8 \pm 1.7$ & $100.0 \pm 2.4$ & $100.0 \pm 4.6$ \\
0.1 & $62.5 \pm 1.4$ & $87.1 \pm 3.2$ & $91.0 \pm 7.2$ \\
1.0 & $49.2 \pm 0.6$ & $68.5 \pm 1.7$ & $84.1 \pm 4.7$ \\
3.0 & $37.4 \pm 0.6$ & $52.1 \pm 2.1$ & $58.7 \pm 3.5$ \\
7.5 & $39.8 \pm 0.5$ & $55.5 \pm 1.9$ & $34.8 \pm 5.6$ \\
\hline
\end{tabular}

EZH2 quantification by ELISA assay. Quantitative detection of EZH2 amount in nuclear extracts of Mino cells was done by enzyme-linked immune sorbent assay (ELISA) using the Human EZH2 (Enhancer of zeste homolog 2) kit (XpressBio, Frederick, MD, USA). AntiEZH2 antibody was pre-coated onto 96-well plates by the manufacturer. Standard concentrations of human EZH2 $(2 \mu \mathrm{g} / \mathrm{ml}-$ $31.25 \mathrm{pg} / \mathrm{ml}$ ), or $10 \mu \mathrm{g}$ of NE were added into each well. For the detection of EZH, a biotin-conjugated anti-EZH2 antibody was used. The concentration of EZH 2 was calculated in $\mathrm{pg} / \mathrm{ml}$ from the standard curve measured at $\mathrm{OD}_{450}$. The half maximal inhibitory concentration $\left(\mathrm{IC}_{50}\right)$ was obtained from linear regression analysis of the concentration-response curves plotted. All data are given as the mean of four independent experiments \pm SD.

Statistical analysis. Student's $t$-test was used to analyze the difference between control and WL-treated samples. Statistical significance was determined at $p<0.05$.

\section{Results}

WL exhibited cytotoxicity against Mino cell line. Mino cells were treated with WL for $24 \mathrm{~h}$ and cell proliferation was measured by WST- 8 assay. WL displayed a dose- dependent inhibition of cell viability with $\mathrm{IC}_{50}$ value of $5.6 \mu \mathrm{M}(p<0.05)$ in comparison to untreated cells in WST-8 proliferation assay.

WL inhibited histone H3K27 methyltransferase activity in NEs from Mino cells. HMT activity was measured in NEs from Mino cells, which were exposed to WL at concentrations ranging from $0.1 \mu \mathrm{M}$ to $10 \mu \mathrm{M}$ in reaction well for $1 \mathrm{~h}$. Results revealed that WL inhibited HMT activity for H3K27 methylation, in a dose-dependent manner (Figure 1) with an $\mathrm{IC}_{50}$ value $\geq 10 \mu \mathrm{M}$ (no statistical significance was found).

WL inhibited histone H3K27 methyltrasferase activity in Mino cells. HMT activity was measured in NEs isolated from Mino cells, which were grown for $24 \mathrm{~h}$ with WL at a range of concentrations from $0.1 \mu \mathrm{M}$ to $7.5 \mu \mathrm{M}$ (Figure 2). WL treatment inhibited HMT activity targeted to H3K27 in 


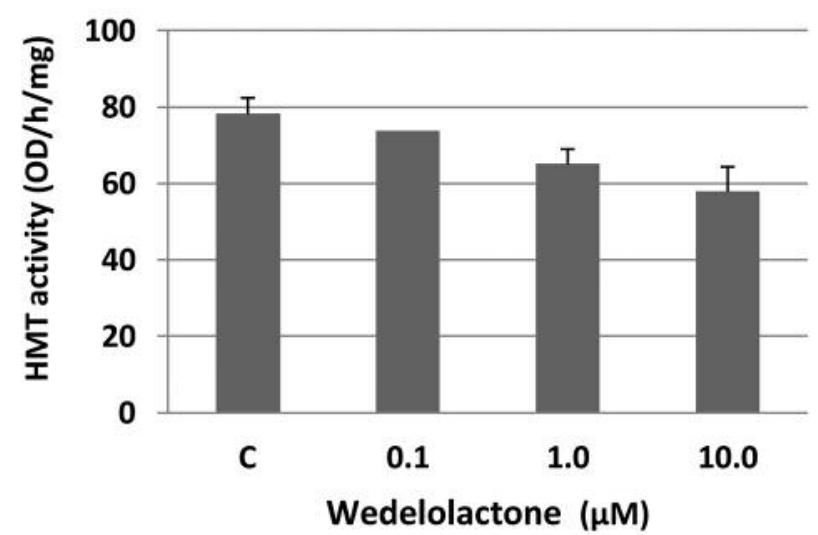

Figure 1. Histone methyltransferase (HMT) activity for methylation of histone $H 3$ at lysine 27 in nuclear extracts (NE) from Mino cells exposed in reaction with different concentrations of Wedelolactone, compared to untreated $N E(C)$. The data are presented as mean $\pm S D$. No statistical significance was found.

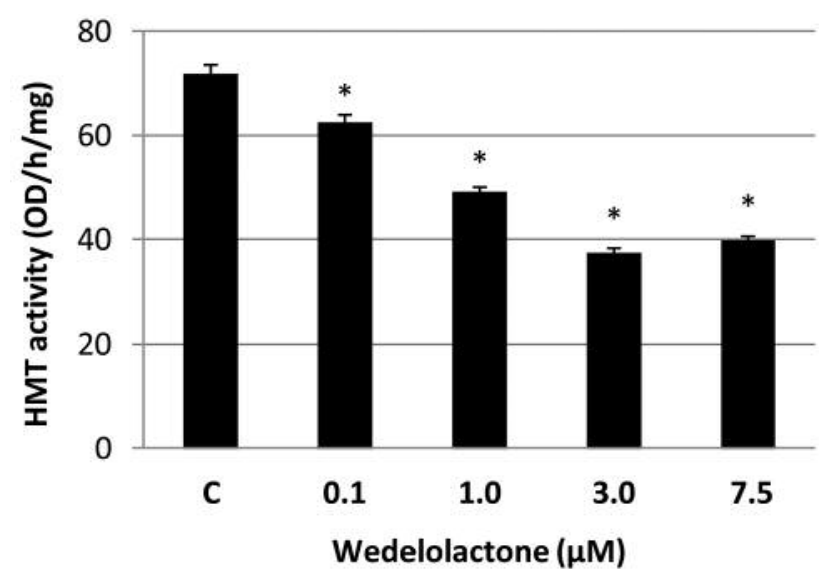

Figure 2. Histone methyltransferase (HMT) activity for methylation of histone $H 3$ at lysine 27 in nuclear extracts (NE) from Mino cells cultivated with different concentrations of Wedelolactone for $24 \mathrm{~h}$ compared to untreated $N E(C)$. The data are presented as mean $\pm S D$. $* p<0.05$ (compared to control).

a dose-dependent manner, with an $\mathrm{IC}_{50}$ value of $3.2 \mu \mathrm{M}$ $(p<0.05)$ in Mino cells. Thus, WL inhibitory effect showed a 3-fold increase in NE from treated cells in comparison to the experiments described above, in which NEs were exposed to WL. This effect could be explained by the suppressive impact of WL on EZH2 expression in Mino cells, which resulted in dose-dependent inhibition of histone H3K27 methylation and Mino cell death (Table I).

WL suppressed H3K27 methylation activity of PRC2 complex in vitro. The H3K27 methylation activity of EZH2, in the

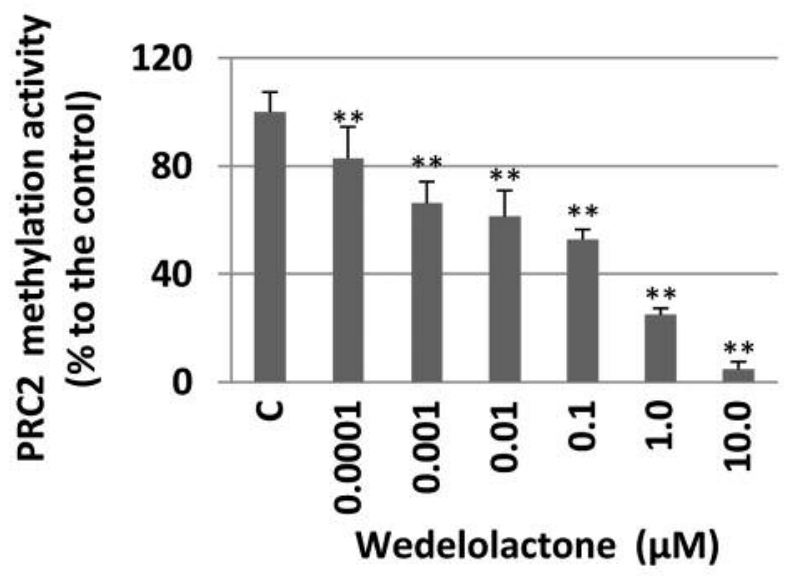

Figure 3. Percentage of histone 3 lysine 27 (H3K27) methylation activity of the PRC2 complex (EZH2/EED/SUZ12/RbAp48/AEBP) exposed in reaction with or without Wedelolactone in control $(C)$. The data are presented as mean $\sigma \pm S D . * * p<0.01$ (compared to control).

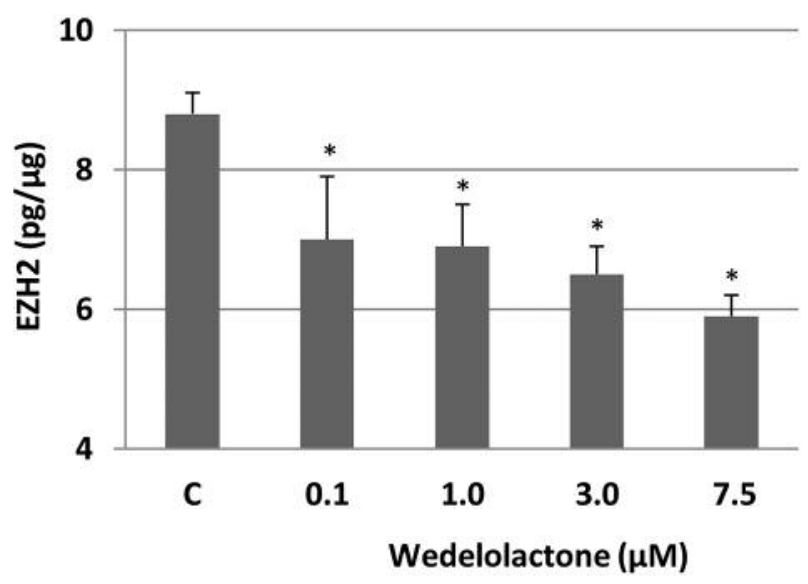

Figure 4. EZH2 amount measured in nuclear extracts from Mino cells treated with Wedelolactone $(0.1,1.0,3.0$, and $7.5 \mu M)$ or untreated $(C)$. The data are presented as mean $\pm S D . * p<0.05$ (compared to control).

context of complete EZH2/EED/SUZ12/RbAp48/AEBP or reduced EZH2/EED/SUZ12 PRC2 complex, was measured in Mino cells with/ without WL treatment, in vitro. Our results revealed that $\mathrm{WL}$, at a range of WL concentrations between $1 \mathrm{nM}$ and $10 \mu \mathrm{M}$, inhibited $\mathrm{H} 3 \mathrm{~K} 27$ methylation activity of PRC2 complex (Figure 3), with an $\mathrm{IC}_{50}$ value of $0.3 \mu \mathrm{M}(p<0.01)$. The inhibitory effect of WL on the H3K27-targeted methylation activity of the reduced PRC2 complex was detected with an $\mathrm{IC}_{50}$ of $1.4 \mu \mathrm{M}(p<0.05)$. Therefore, WL suppressed EZH2 activity in the complete PRC2 complex 3-fold more efficient than in the reduced 
PRC2 complex. Thus, removal of cofactor proteins RbAp48/AEBP from PRC2 complex decreased WL activity towards inhibition of histone H3K27 methylation.

WL down-regulated the expression of EZH2 in Mino cells. Together with the HMT activity we measured the EZH2 amount in NEs from Mino cells treated with WL at concentrations of $0.1,1,3$, and $7.5 \mu \mathrm{M}$, for $24 \mathrm{~h}$. ELISA assay showed that WL decreased EZH2 quantity in a dosedependent manner in Mino cells (Figure 4). More specifically, maximum EZH2 amount was decreased from $8.8 \pm 0.3 \mathrm{pg} / \mu \mathrm{g}$ in untreated Mino cells to $5.9 \pm 0.3 \mathrm{pg} / \mu \mathrm{g}$ $(p<0.05)$ in WL- treated cells with $7.5 \mu \mathrm{M}$ concentration.

\section{Discussion}

Natural products targeting EZH2 are considered as promising agents for the prevention and treatment of cancer. Several natural products, such as gambogic acid, triptolide, curcumin, and ursolic acid, have been shown to serve as EZH2 modulators (14). There are still no data about the epigenetic regulation of EZH2-mediated catalysis of H3K27 methylation in MCL. The present study was focused on the effects of WL, a plant coumestan that occurs in Wedelia calendulacea, on H3K27 methylation in the MCL cell line, Mino.

A summary of the effects of WL on the EZH2 amount, HMT activity, and cell proliferation in Mino cells, is presented in Figure 5. Specifically, WL inhibited the proliferation of Mino cells. Moreover, down-regulated HMT activity was detected in NEs directly exposed to WL, as well as in NEs from MCL cultivated with WL. We supposed that downregulated expression of EZH2 in WL-treated Mino cells could entail suppression of HMT activity. Indeed, EZH2 amount was decreased in WL-treated cells. Thus, we showed two different plausible mechanisms of WL impact on MCL. First, WL inhibited the proliferation of Mino cells (Figure 5). Second, WL suppressed methylation of histone 3 at lysine 27 through down-regulation of EZH2 amount and subsequent reduction of HMT activity. To determine the exact mechanism of WL action additional experimental studies would be required.

PRC2 is an important epigenetic regulator. It is involved in the transcriptional regulation of target genes, it is responsible for the H3K27 methylation, and plays an important role in tumorigenesis by silencing tumor suppressor genes (23). However, natural compounds targeting the EZH2 to disable PRC2 complex are scarcely described. It was recently shown that WL disrupts the interaction of EZH2 and EED components in vitro and induces the degradation of PRC2 complex, resulting in cancer cell growth arrest (15). Moreover, it was shown that WL acts as an anti-cancer agent for breast and prostate carcinomas, in vitro and in vivo (24). Herein, it was demonstrated that absence of the RbAp48 and AEBP proteins in the core enzymatic subunit EZH2/EED/SUZ12 of

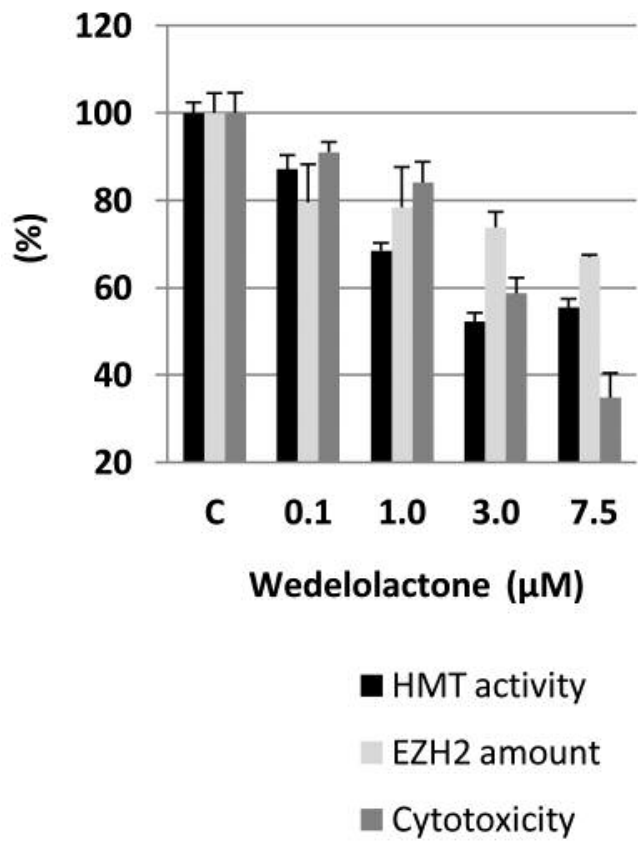

Figure 5. Summary of the effects of Wedelolactone treatment on histone 3 lysine 27 (H3K27) histone methyltransferase (HMT) activity, EZH2 amount, and cytotoxicity (in percent) in Mino cells treated with 0.1, 1.0, 3.0, and $7.5 \mu \mathrm{M}$ of Wedelolactone in comparison to untreated cells $(C)$. The data are presented as mean $\pm S D$.

the PRC2 complex decreased of the inhibitory activity of WL on H3K27 methylation. Thus, we proposed that WL inhibited EZH2 methylation activity in MCL in the case of complete PRC2 complex. The functions of the PRC2 complex cofactors are not still completely clear. Ciferri and co-workers have revealed that AEBP protein interacts with EZH2 and SUZ12 in the PRC2 complex to enhance EZH2 enzymatic activity (25). Although they are not required for the EZH2-modulated HMT activity, the RbAp46/48proteins have been shown to possess an important PRC2-related function such as chaperone proteins that bind to histone $\mathrm{H} 3-\mathrm{H} 4$ and plays a pivotal role in the assembly of nucleosomes $(26,27)$.

In conclusion, our results suggested $\mathrm{WL}$ as a promising agent for the treatment of MCL and provided a plausible mechanism for the presumable anticancer effects. Taken together, down-regulation of EZH2 expression in Mino cells treated with WL was combined with the inhibition of cell proliferation and suppression of histone methyltransferase activity in vitro and WL-treated cells. Thus, WL could serve as a candidate for the therapy of MCL.

\section{Authors' Contributions}

Romanchikova N. designed and performed experiments, analyzed data, wrote the paper and provided final approval of the version to 
publish. Trapencieris P. generated ideas, made literature analysis, and contributed to the manuscript editing.

\section{Conflicts of Interest}

The Authors declare that there is no conflict of interests.

\section{Acknowledgements}

This project was supported by IG-2018-12 grant of Latvian Institute of Organic Synthesis.

\section{References}

1 Neff T, Sinha AU, Kluk MJ, Zhu N, Khattab MH, Stein L, Xie $\mathrm{HF}$, Orkin SH and Armstrong SA: Polycomb repressive complex 2 is required for MLL-AF9 leukemia. PNAS USA 109: 50285033, 2012. PMID: 22396593. DOI: 10.1073/pnas.1202258109

2 Li Z, Wang Y, Qiu J, Li Q, Yuan C, Zhang W, Wang D Ye J, Jiang $\mathrm{H}$, Yang $\mathbf{J}$ and Cheng $\mathrm{J}$ : The polycomb group protein EZH2 is a novel therapeutic target in tongue cancer. Oncotarget 4: 25322549, 2013. PMID: 24345883. DOI: 10.18632/oncotarget.1503

3 Gao SB, Xu B, Ding LH, Zheng QL, Zhang L, Zheng QF, Li SH, Feng ZJ, Wei J, Yin ZY, Hua X and Jin GH: The functional and mechanistic relatedness of EZH2 and menin in hepatocellular carcinoma. J Hepatol 61(4): 832-983, 2014. PMID: 24845612. DOI: 10.1016/j.jhep.2014.05.015

4 Yoo KH and Hennighausen L: EZH2 methyltransferase and H3K27 methylation in breast cancer. Int J Biol Sci 8(1): 59-65, 2012. PMID: 22211105. DOI: 10.7150/ijbs.8.59

$5 \mathrm{Kim} \mathrm{KH}$ and Roberts CW: Targeting EZH2 in cancer. Nat Med 22(2): 128-134, 2016. PMID: 26845405. DOI: 10.1038/nm.4036

6 Shin YJ and Kim JH: The role of EZH2 in the regulation of the activity of matrix metalloproteinases in prostate cancer cells. PLoS One7: e30393, 2012. PMID: 22272343. DOI: 10.1371/ journal.pone.0030393

7 Wang CG, Ye YJ, Yuan J, Liu FF, Zhang H and Wang S: EZH2 and STAT6 expression profiles are correlated with colorectal cancer stage and prognosis. World J Gastroenterol 16: 24212427, 2010. PMID: 20480530. DOI: 10.3748/wjg.v16.i19. 2421

8 Neusquen LP, Filassi JR, Fristachi CE, Carvalho KC, Doria MT, Soares Junior JM and Piato JR: EZH2 protein expression and tumor response to neoadjuvant chemotherapy in locally advanced breast cancer. Rev Bras Ginecol Obstet 38(6): 280286, 2016. PMID: 27399922. DOI: 10.1055/s-0036-1584954

9 Sun R, Shen J, Gao Y, Zhou Y, Yu Z, Hornicek F, Kan Q and Duan Z: Overexpression of EZH2 is associated with the poor prognosis in osteosarcoma and function analysis indicates a therapeutic potential. Oncotarget 7: 38333-38346, 2016. PMID: 27223261. DOI: $10.18632 /$ oncotarget.9518

10 Wassef M, Michaud A and Margueron R: Association between EZH2 expression, silencing of tumor suppressors and disease outcome in solid tumors. Cell Cycle 15(17): 2256-2262, 2016. PMID: 27419533. DOI: 10.1080/15384101.2016.1208872

11 Ebert A, Schotta G, Lein S, Kubicek S, Krauss V, Jenuwein T and Reuter G: $\mathrm{Su}$ (var) genes regulate the balance between euchromatin and heterochromatin in Drosophila. Genes Dev 18: 2973-2983, 2004. PMID: 15574598. DOI: $10.1101 / \operatorname{gad} .323-04$
12 Shen X, Liu Y, Hsu YJ, Fujiwara Y, Kim J, Mao X, Yuan GC and Orkin SH: EZH1 mediates methylation on histone H3 lysine 27 and complements EZH2 in maintaining stem cell identity and executing pluripotency. Mol Cell 32(4): 491-502, 2008. PMID: 19026780. DOI: 10.1016/j.molcel.2008.10.016

13 Jacob Y, Feng S, LeBlanc CA, Bernatavichute YV, Stroud H, Cokus S, Johnson LM, Pellegrini M, Jacobsen SE and Michaels SD: ATXR5 and ATXR6 are H3K27 mono- methyltransferases required for chromatin structure and gene silencing. Nat Struct Mo Biol 16(7): 763-768, 2009. PMID: 19503079. DOI: 10.1038/ nsmb.1611

14 Shahabipour F, Caraglia M, Majeed M, Derosa G, Maffioli P and Sahebkar A: Naturally occurring anti-cancer agents targeting EZH2. Cancer Lett 400: 325-335, 2017. PMID: 28323035. DOI: 10.1016/j.canlet.2017.03.020

15 Chen H, Gao T, Li J, Liu D, Sheng C, Yao C, Jiang W, Wu J, Chen $\mathrm{S}$ and Huang W: Wedelolactone disrupts the interaction of EZH2-EED complex and inhibits PRC2-dependent cancer. Oncotarget 6(15): 13049-13059, 2015. PMID: 25944687. DOI: 10.18632/oncotarget. 3790

16 Kobori M, Yang Z, Gong D, Heissmeyer V, Zhu H, Jung YK, Gakidis MA, Rao A, Sekine T, Ikegami F, Yuan C and Yuan J: Wedelolactone suppresses LPS-induced caspase-11 expression by directly inhibiting the IKK complex. Cell Death Differ 11: 123-130, 2004. PMID: 14526390. DOI: 10.1038/sj.cdd.4401325

17 Tsai CH, Tzeng S, Hsieh S, Yang YC, Hsiao Y, Tsai M and Hsiao PW: A standardized herbal extract mitigates tumor inflammation and augments chemotherapy effect of docetaxel in prostate cancer. Sci Rep 7(1): 15624-15628, 2017. PMID: 29142311. DOI: $10.1038 / \mathrm{s} 41598-017-15934-0$

18 Bharadwaj U, Marin-Muller C, Li M, Chen C and Yao Q: Mesothelin overexpression promotes autocrine IL-6/sIL-6R trans-signaling to stimulate pancreatic cancer cell proliferation. Carcinogenesis 32(7): 1013-1024, 2011. PMID: 21515913. DOI: $10.1093 /$ carcin/bgr075

19 Idris AI, Libouban H, Nyangoga H, Landao-Bassonga E, Chappard D and Ralston SH: Pharmacologic inhibitors of IkappaB kinase suppress growth and migration of mammary carcinosarcoma cells in vitro and prevent osteolytic bone metastasis in vivo. Mol Cancer Ther 8(8): 2339-2347, 2009. PMID: 19671767. DOI: 10.1158/1535-7163.MCT-09-0133

20 Hsieh CJ, Kuo PL, Hou MF, Hung JY, Chang FR, Hsu YC, Huang YF, Tsai EM and Hsu YL: Wedelolactone inhibits breast cancer-induced osteoclastogenesis by decreasing Akt/mTOR signaling. Int J Oncol 46(2): 555-562, 2015. PMID: 25421824. DOI: $10.3892 /$ ijo.2014.2769

21 Hershkovitz-Rokah O, Pulver D, Lenz G and Shpilberg O: Ibrutinib resistance mantle cell lymphoma clinical, molecular and treatment aspects. Br J Hematol 181(3): 306-319, 2018. PMID: 29359797. DOI: $10.1111 / \mathrm{bjh} .15108$

22 Romanchikova N, Strods A, Strazdina J, Strumfs B and Trapencieris P: A novel aziridine-based Bruton's tyrosine kinase inhibitor induces apoptosis through down-regulation of p65/RelA phosphorylation on serine 536 and ERK1/2 in mantle cell lymphoma. Anticancer Res 36(11): 6133-6140, 2016. PMID: 27793942. DOI: 10.21873/anticanres.11204

23 Zingg D, Debbache J, Schaefer SM, Tuncer E, Frommel SC, Cheng P, Arenas-Ramirez N, Haeusel J, Zhang Y, Bonalli M, McCabe MT, Creasy CL, Levesque MP, Boyman O, Santoro R, Shakhova O, Dummer R and Sommer L: The epigenetic modifier 
EZH2 controls melanoma growth and metastasis through silencing of distinct tumour suppressors. Nat Commun 6: 6051-6067, 2015. PMID: 25609585. DOI: 10.1038/ncomms 7051

24 Nehybova T, Smarda J, Daniel L, Brezovsky J and Benes P: Wedelolactone induces growth of breast cancer cells by stimulation of estrogen receptor signaling. J Steroid Biochem Mol Biol 152: 76-83, 2015. PMID: 25934092. DOI: 10.1016/ j.jsbmb.2015.04.019

25 Ciferri C, Lander GC, Maiolica A, Herzog F, Aebersold R and Nogales E: Molecular architecture of human polycomb repressive complex 2. eLife 1: e00005, 2012. PMID: 23110252. DOI: $10.7554 /$ eLife.00005

26 Nekrasov M, Wild B and Muller J: Nucleosome binding and histone methyl-transferase activity of Drosophila PRC2. EMBO Rep 6(4): 348-353, 2005. PMID: 15776017. DOI: 10.1038/ sj.embor.7400376
27 Zhang W, Tyl M, Ward R, Sobott F, Maman J, Murthy AS, Watson AA, Fedorov O, Bowman A, Owen-Hughes T, El Mkami $\mathrm{H}$, Murzina NV, Norman DG and Laue ED: Structural plasticity of histones $\mathrm{H} 3-\mathrm{H} 4$ facilitates their allosteric exchange between RbAp48 and ASF1. Nat Struct Mol Biol 20(1): 29-35, 2013. PMID: 23178455. DOI: $10.1038 / \mathrm{nsmb} .2446$

Received May 24, 2019

Revised June 20, 2019

Accepted June 24, 2019 